\title{
Desafíos y experiencias de administradores escolares novatos en el sureste de México
}

\author{
Challenges and Experiences of Novel School Administrators from Southeast \\ México
}

\section{Desafios e experiências de administradores de escolas iniciantes no sudeste do México}

Edith J. Cisneros-Cohernour

Universidad Autónoma de Yucatán, México

ecohernour@gmail.com

https://orcid.org/0000-0003-2319-1519

\section{Resumen}

El propósito de este estudio fue identificar los desafíos que enfrentan directores de educación primaria en los primeros años de su gestión y en qué medida consideran que su preparación previa los ayudó para enfrentarlos. El estudio fue descriptivo. Incluyó una encuesta que se aplicó a 127 directores de los estados de Yucatán, Campeche y Quintana Roo. Para la recolección de datos se utilizó el cuestionario desarrollado por Webber (2008). Los resultados indican que existen diferencias significativas entre los retos de los directores de los tres estados, aunque no existieron diferencias en relación con los siguientes desafíos: iniciar y dar continuidad a proyectos de mejora escolar, lidiar con personal académico y administrativo con bajo desempeño, mejorar las capacidades profesionales de los profesores, manejar conflictos, lograr un equilibrio entre su vida personal y familiar, aplicar la normatividad, lidiar con papeleo administrativo y tener acceso a las autoridades del sistema escolar. Asimismo, se encontró que la gran mayoría de los directores en los tres estados son hombres. Los directores perciben que su formación docente previa es valiosa para el ejercicio de su papel como directores, pero indicaron requerir formación en gestión escolar, normatividad, pedagogía, psicología, orientación, así como en tecnologías de información y comunicación. 


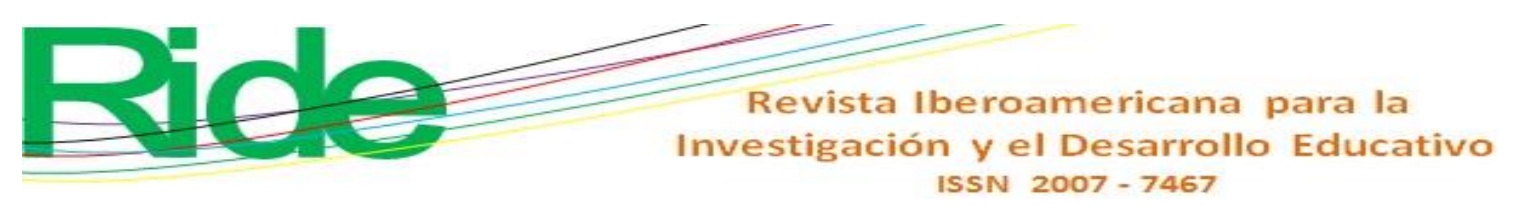

Palabras clave: administración de la educación, enseñanza primaria, liderazgo, México.

\begin{abstract}
The purpose of this study was to identify the challenges faced by elementary school principals during the first years of their administration and the extent to which they consider that their previous preparation helped them to face them. The study was descriptive, a survey carried out with one hundred and twenty-seven principals from the states of Yucatán, Campeche, and Quintana Roo, Mexico. During data collection, the questionnaire developed by Webber (2008) was used. Findings indicate that there were significant differences between the challenges that the principals face in the three states, although there were no differences about to with concerning the following challenges: initiating and continuing school improvement projects, dealing with underperforming academic and administrative staff, improving the professional capacities of teachers, handling conflicts, achieving a balance between personal and family life, enforcing regulations, dealing with administrative paperwork and having access to the authorities of the school system. Likewise, most principals in the three states are men. The participants perceive that their previous teaching training is valuable for exercising their role as principals, but they indicated to require training in school management, regulations, pedagogy, psychology, guidance, as well as in information and communication technologies.
\end{abstract}

Keywords: educational administration, primary education, leadership, Mexico.

\title{
Resumo
}

O objetivo deste estudo foi identificar os desafios enfrentados pelos dirigentes do ensino fundamental nos primeiros anos de sua gestão e em que medida consideram que a sua preparação anterior os ajudou a enfrentá-los. O estudo foi descritivo. Incluiu uma pesquisa aplicada a 127 diretores dos estados de Yucatán, Campeche e Quintana Roo. Para a coleta de dados, foi utilizado o questionário desenvolvido por Webber (2008). Os resultados indicam que existem diferenças significativas entre os desafios dos dirigentes dos três estados, embora não tenham ocorrido diferenças em relação aos seguintes desafios: iniciar e dar continuidade a projetos de melhoria escolar, lidar com pessoal acadêmico e administrativo de baixo desempenho, melhorar as capacidades profissionais de professores, lidar com conflitos, alcançar um equilíbrio entre a vida pessoal e familiar, fazer cumprir os regulamentos, lidar 


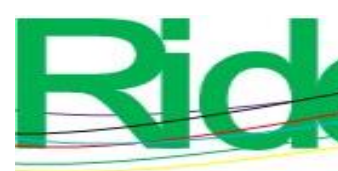

Revista Iberoamericana para la
Investigación y el Desarrollo Educativo
ISSN $2007-7467$

com a papelada administrativa e ter acesso às autoridades do sistema escolar. Da mesma forma, constatou-se que a grande maioria dos conselheiros nos três estados são homens. Os diretores percebem que sua formação docente anterior é valiosa para o exercício de sua função de diretores, mas indicaram que requerem formação em gestão escolar, regulamentação, pedagogia, psicologia, orientação, bem como em tecnologias de informação e comunicação.

Palavras-chave: administração educacional, educação primária, liderança, México.

Fecha Recepción: Julio 2021

Fecha Aceptación: Enero 2022

\section{Introducción}

La investigación sobre las experiencias y problemas de quienes ejercen la dirección escolar en sus primeros años de gestión es relativamente reciente y aún requiere mucha más atención, especialmente por el importante papel del liderazgo de los directores en los resultados de aprendizaje y en la efectividad de la organización.

Entre los estudios que han encontrado que el ejercicio del liderazgo del director influye en los resultados de aprendizaje de los estudiantes, se encuentra el trabajo de Hallinger y Heck (1998), quienes determinaron que la gestión del director tiene un efecto indirecto sobre variables intervinientes que afectan el aprendizaje. Igualmente, los estudios de Day, Leithwood y Sammons (2008), Day, Sammons, Hopkins, Leithwood y Kington (2008), Leithwood y Day (2008), Leithwood, Harris y Hopkins (2008) y Gu, Sammons y Mehta (2008) han confirmado el efecto del liderazgo del director en la docencia.

De acuerdo con Leithwood et al. (2008), el liderazgo es superado solo por la influencia de la docencia en el aprendizaje de los alumnos. Cuando se incluyen tanto las variables directas como las indirectas, el liderazgo puede explicar $25 \%$ del rendimiento de los estudiantes. Los líderes ejercen esta influencia de manera indirecta a través de la motivación de los docentes, el compromiso y las condiciones laborales. Es importante notar que los autores también encontraron que el tipo de liderazgo que tiene mayor influencia es el que está ampliamente distribuido, esto es, cuando se favorece el liderazgo de los otros actores en la comunidad escolar (Harris, 2003; Webber y Okoko, 2021).

Rice (2010), quien revisó la literatura sobre este tema, concluyó: 


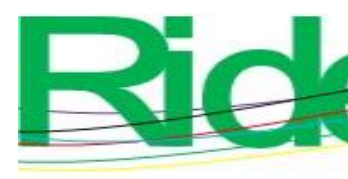

Revista Iberoamericana para la
Investigación y el Desarrollo Educativo
ISSN $2007-7467$

Los retos que suponen los aspectos administrativos del puesto son ciertamente un tema importante. Por ejemplo, se ha encontrado que los directores novatos suelen enfrentar el reto de la rendición de cuentas, por lo que los programas de preparación de directores deberían considerar este aspecto en su desarrollo (Shirrell, 2016). Otro reto relacionado es la dificultad para poder lidiar contra las exigencias de actores externos a la institución educativa, en especial cuando se requieren tomar acciones poco populares (Prado y Spillane, 2019).

Otras barreras que impiden una transición suave en el puesto director incluyen un equipo de trabajo pobre, relaciones tensas con el personal y la dificultad para convencer el equipo de la visión del director (Arar, 2018; Marshall, 2018), así como la discriminación que puede verse reflejada en el proceso de selección, donde las mujeres y otros grupos minoritarios suelen estar subrepresentados en los procesos de elección de directores nuevos (Lee y Mao, 2020).

El puesto de dirección, además, puede conllevar para los nuevos directores una falta de apoyo profesional y emocional (Arar, 2018). De manera reciente, algunos estudios han examinado la soledad y el aislamiento que genera este cargo (Arar, 2018; Bauer y Silver, 2018; Tahir, Thakib, Hamzah, Said y Musah, 2017). Si bien se trata de emociones relativamente poco frecuentes y temporales y usualmente asociadas a una poca voluntad para compartir sus conocimientos con el resto de los docentes o a una mala comunicación con estos (Tahir et al., 2017), los análisis señalan que están asociadas de manera negativa con la autoeficacia, la satisfacción laboral y de manera positiva con el desgaste profesional y de manera fuerte con la intención de renunciar (Bauer y Silver, 2018; DeMatthews, Reyes, Carrola, Edwards y James, 2021). Este último par deja de manifiesto la necesidad de que el director principiante desarrolle adecuadas estrategias de afrontamiento que le permitan sobrellevar los retos del puesto (DeMatthews et al., 2021).

Por otro lado, también se han identificado algunos elementos que pueden facilitar la transición al nuevo puesto directivo. Por ejemplo, algunos facilitadores en la transición al puesto de dirección incluyen mentorías con directores previos (Arar, 2018; Marshall, 2018; Oplatka y Lapidot, 2018), experiencia previa en puestos de liderazgo y un equipo de trabajo cooperativo (Marshall, 2018). La motivación es otro facilitador importante. Al respecto, es usual que los directores principiantes manejen un discurso de vocación para encauzar sus motivaciones en el puesto, en particular en contexto desafiantes, ya que lo describen como 


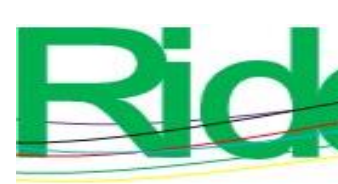

Revista Iberoamericana para la
Investigación y el Desarrollo Educativo
ISSN $2007-7467$

Lo anterior, más los retos identificados en las investigaciones previamente señaladas, han conllevado a proponer algunos elementos para mejorar el proceso de formación de los nuevos directores. No es de extrañar que algunas propuestas de preparación de directores sistematicen el proceso en cuatro grandes áreas, cuyo nombre puede ir variando de acuerdo con el autor: contextualización del lugar/sistema, el desarrollo de las relaciones con el equipo de trabajo, el sistema administrativo y el autocuidado y bienestar de los directores (Slater et al., 2018).

En particular, las mentorías han demostrado jugar un papel relevante en la formación de directores principiantes. Los mentores brindan a los nuevos directores retroalimentación sobre su desempeño y consejos sobre el manejo de actividades administrativas más que de liderazgo, así como apoyo emocional (Oplatka y Lapidot, 2018; Viloria et al., 2021). Los programas de mentorías para directores ofrecen, además, un espacio "seguro" que genera un mayor sentimiento de empoderamiento en los nuevos directores (Simon, Dole y Farragher, 2019). Cabe señalar que, a pesar de que han sido recomendados para mejorar la disparidad de género en los puestos de directores, estos continúan siendo raros para los casos de las mujeres (Lee y Mao, 2020).

Por su parte, el rol del director principiante en la mejora escolar es el tercer tema más abordado, haciendo especial énfasis en aspectos de liderazgo instruccional o la evaluación de su cuerpo docente (Kılınç y Gümüş, 2021).

El desarrollo del liderazgo en los directores principiantes es un ejercicio de interacción entre la preparación recibida y la socialización del puesto que les permite comprender los aspectos organizacionales del puesto (Murphy, 2020).

El logro de los objetivos propuestos por los directores nuevos y su éxito se asocia con su compromiso, esfuerzo, aprendizaje y con el apoyo del servicio (Meyer, Sinnema y Patuawa, 2019). Como se ha visto, es otro facilitador importante, especialmente cuando los nuevos directores visualizan su puesto en términos de vocación (Swen, 2020).

\section{Investigaciones realizadas en México}

En México, se asigna un director de educación primaria a un centro escolar por medio del escalafón, un sistema de puntos basado en años de experiencia, cursos y evaluación de la enseñanza. Los principales actores del centro escolar no son consultados sobre quién debe ser designado para el puesto. El director o directora no se reúne con los padres, maestros y 


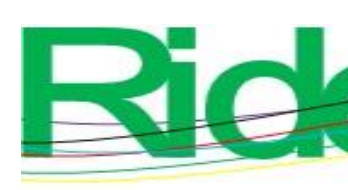

Revista Iberoamericana para la
Investigación y el Desarrollo Educativo
ISSN $2007-7467$

estudiantes sino hasta el primer día de trabajo, ya que en la mayor parte de los casos son asignados a otros centros escolares. Asimismo, la mayoría de las escuelas primarias en México están asignadas a un doble turno. Hay un turno de mañana y otro de tarde en el mismo edificio de la escuela.

En relación con las investigaciones, se han realizado algunos estudios, principalmente a raíz de la implementación del Proyecto Escolar (Secretaría de Educación Pública [SEP], 1999) y el Programa Escuelas de Calidad (PEC). Ruiz (1999) encontró que el papel de los directores tenía una correlación relativamente fuerte con el rendimiento de los estudiantes. Loera, Hernández y García (2006) estudiaron el liderazgo escolar como parte de la evaluación del PEC y demostraron que el director marcó una diferencia en la mejora del rendimiento estudiantil. Camarillo (2006) analizó factores de éxito y fracaso en Proyecto Escolar, y concluyó que el director es fundamental para el éxito de este.

Asimismo, en varios estados mexicanos se realizaron estudios de caso como parte del International Study of Principal Preparation (ISPP), una investigación sobre los retos de administradores escolares noveles en 10 países (Onguko et al., 2008, 2012; Webber, 2008). El proyecto se centró en la utilidad de la preparación recibida para el puesto desde la perspectiva de los directores en sus primeros tres años en el cargo. Entre los resultados de estos estudios de caso, se encuentran los siguientes: en la Ciudad de México, Slater, Esparza et al. (2005) reportaron dos desafíos importantes de los directores de escuela. El primero fue la falta de recursos; el otro fue cómo motivar a aquellos con quienes trabajaban: maestros, personal y supervisores. Los directores expresaron su frustración con los maestros que no compartieron su dedicación.

En su análisis, Slater, Boone et al. (2005) encontraron que los directores de escuelas abordaron sus puestos administrativos con una de tres predisposiciones, ya sea buscando mejorar las condiciones de educación (agente de cambio), promoverse (ambición) o adquirir competencias personales (superación personal). Muchos de estos directores de escuela provenían de la enseñanza en el aula. Se veían a sí mismos como agentes de cambio ambiciosos que tenían los mejores intereses para su escuela y la nación, pero puede que esta no sea la perspectiva que los maestros tenían de ellos.

En Baja California, Slater, Esparza et al. (2005) estudiaron a 15 directores de escuelas en áreas rurales. Expresaron preocupaciones similares sobre la actitud de los profesores y sus frecuentes ausencias. Los directores estaban ansiosos por hablar sobre sus luchas en comunidades con problemas de pobreza, drogadicción y fugacidad de las familias. 


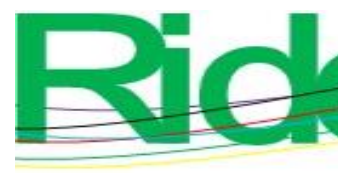

Revista Iberoamericana para la
Investigación y el Desarrollo Educativo
ISSN $2007-7467$

En los estudios de caso antes mencionados se encontró que algunos directores se quejaron de la falta de dedicación de los profesores, que a menudo se expresaba en términos de puntualidad. Querían que los maestros llegaran a tiempo, trabajaran en colaboración y se comunicaran con los padres. Algunos dijeron que el sindicato protegía a los maestros que no querían trabajar duro.

Los directores tenían una enorme cantidad de papeleo, pero tenían poca capacitación en las leyes, reglas, regulaciones y políticas que se suponía que debían implementar e informar (Slater, García y Gorosave, 2007). Les preocupaba que los padres no apoyaran vigorosamente la misión de la escuela, no quisieran asistir a la escuela ni ofrecerse como voluntarios para ayudar. Algunos padres preferían que sus hijos se quedaran en casa para ayudar con las tareas del hogar.

Por último, en el estado de Sonora, Slater, Boone et al. (2007) encontraron que los directores enfrentaron desafíos adicionales en su primer año en relación con el sistema de designación al puesto y la estructura de la jornada escolar.

\section{Propósito del estudio}

Dada la necesidad de examinar si los resultados de estos estudios son consistentes con las necesidades de formación de los directores escolares en otras regiones de México, esta investigación tuvo como propósito identificar los desafíos que enfrentan directores de educación primaria en los primeros años de su gestión y la medida en que consideran que su bagaje previo los preparó para enfrentarlos.

\section{Método}

Este estudio fue cuantitativo, de tipo descriptivo, ya que, como indican Gall, Gall y Borg (2007), se centra en describir los fenómenos y sus características, más que en examinar por qué tienen lugar. El estudio descriptivo se realizó por medio de una encuesta a los directores de tres estados del sureste mexicano. 


\section{Participantes}

En colaboración con la Secretaría de Educación del Gobierno de los estados de Yucatán, Campeche y Quintana Roo, se identificaron a los 130 directores, de los cuales se obtuvo una participación de 127. Las características demográficas de los participantes se presentan en la tabla 1 .

Tabla 1. Datos y características sociodemográficos de los participantes

\begin{tabular}{|c|c|c|c|}
\hline Variable & $\begin{array}{l}\text { Yucatán } \\
(N=46)\end{array}$ & $\begin{array}{l}\text { Campeche } \\
(N=46)\end{array}$ & $\begin{array}{c}\text { Quintana Roo } \\
(N=35)\end{array}$ \\
\hline \multicolumn{4}{|l|}{ Sexo } \\
\hline Masculino & $59.0 \%$ & $80.0 \%$ & $63.0 \%$ \\
\hline Femenino & $41.0 \%$ & $18.0 \%$ & $37.0 \%$ \\
\hline \multicolumn{4}{|l|}{ Edad } \\
\hline Menos de 25 años & $0.0 \%$ & $1.1 \%$ & $0.0 \%$ \\
\hline 25 a 34 años & $3.0 \%$ & $20.2 \%$ & $11.4 \%$ \\
\hline 35 a 44 años & $10.0 \%$ & $41.5 \%$ & $31.4 \%$ \\
\hline 45 a 54 años & $30.0 \%$ & $28.7 \%$ & $57.1 \%$ \\
\hline 55 o más años & $3.0 \%$ & $7.4 \%$ & $0.0 \%$ \\
\hline \multicolumn{4}{|l|}{ Grado académico } \\
\hline Normal básica & $4.0 \%$ & $19.1 \%$ & $34.3 \%$ \\
\hline Licenciatura & $29.0 \%$ & $68.1 \%$ & $54.3 \%$ \\
\hline Estudios de maestría & $2.0 \%$ & $8.5 \%$ & $5.7 \%$ \\
\hline Grado de maestría & $8.0 \%$ & $3.2 \%$ & $2.9 \%$ \\
\hline Estudios de doctorado & $3.0 \%$ & $1.1 \%$ & $0.0 \%$ \\
\hline Grado de doctorado & $0.0 \%$ & $0.0 \%$ & $0.0 \%$ \\
\hline
\end{tabular}

Fuente: Elaboración propia

Como se advierte en la tabla anterior, la mayor parte de los directores son hombres, especialmente en caso del estado de Campeche. En cuanto a edad, la mayor parte tiene entre 45 y 50 años, con excepción del estado de Campeche, donde la mayoría tiene entre 35 y 44 años. En relación con el máximo grado de estudios, la mayor parte tiene estudios de licenciatura. 


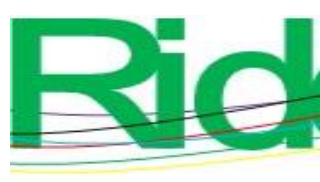

Revista Iberoamericana para la Investigación y el Desarrollo Educativo ISSN 2007 - 7467

Las características de los directores en relación con su experiencia docente y profesional se presentan en la tabla 2.

Tabla 2. Características de su experiencia en el puesto directivo de los participantes

\begin{tabular}{|c|c|c|c|}
\hline Variable & $\begin{array}{l}\text { Yucatán } \\
(N=46)\end{array}$ & $\begin{array}{l}\text { Campeche } \\
(N=46)\end{array}$ & $\begin{array}{l}\text { Quintana Roo } \\
\qquad(N=35)\end{array}$ \\
\hline \multicolumn{4}{|l|}{$\begin{array}{l}\text { Años completos en el puesto de } \\
\text { director(a) }\end{array}$} \\
\hline Un año & $16.0 \%$ & $29.8 \%$ & $60.0 \%$ \\
\hline Dos años & $16.0 \%$ & $18.1 \%$ & $14.3 \%$ \\
\hline Tres años & $12.0 \%$ & $37.2 \%$ & $14.3 \%$ \\
\hline Cuatro años & $0.0 \%$ & $2.1 \%$ & $0.0 \%$ \\
\hline \multicolumn{4}{|l|}{$\begin{array}{l}\text { Años de experiencia profesional en } \\
\text { un puesto formal administrativo o de } \\
\text { liderazgo antes de su puesto actual } \\
\text { como director de primaria }\end{array}$} \\
\hline Menos de cinco años & $19.0 \%$ & $37.1 \%$ & $37.1 \%$ \\
\hline Cinco a nueve años & $6.0 \%$ & $8.6 \%$ & $8.6 \%$ \\
\hline 10 a 19 años & $2.0 \%$ & $5.7 \%$ & $5.7 \%$ \\
\hline 20 años y más & $6.0 \%$ & $2.9 \%$ & $2.9 \%$ \\
\hline \multicolumn{4}{|l|}{$\begin{array}{l}\text { Número total de años de experiencia } \\
\text { profesional en educación, antes de } \\
\text { ocupar el puesto actual }\end{array}$} \\
\hline Menos de cinco años & $2.0 \%$ & $40.4 \%$ & $0.0 \%$ \\
\hline Cinco a nueve años & $6.0 \%$ & $17.0 \%$ & $5.7 \%$ \\
\hline 10 a 19 años & $7.0 \%$ & $7.4 \%$ & $34.3 \%$ \\
\hline 20 años y más & $31.0 \%$ & $6.4 \%$ & $60.0 \%$ \\
\hline
\end{tabular}

Fuente: Elaboración propia

La mayor parte de los participantes se encontraba en su primer año como directores, algunos tenían experiencia en puestos administrativos, pero por corto tiempo. En relación con su experiencia en educación, los directores de Yucatán y Quintana Roo reportaron tener 


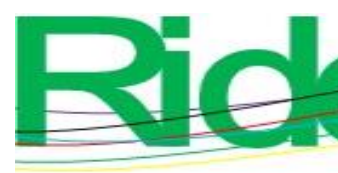

Revista Iberoamericana para la
Investigación y el Desarrollo Educativo
ISSN $2007-7467$

mayor número de años de experiencia profesional en comparación con sus colegas de Campeche.

Las escuelas en las que trabajan los directores tienen diferentes características. En el caso del estado de Campeche, $43.6 \%$ de las escuelas tiene menos de 100 estudiantes, y $25 \%$ tiene entre 200 a 300 estudiantes. Por su parte, solo $45 \%$ de los directores de Yucatán, respondieron a esta pregunta, entre estos $34 \%$ tiene escuelas entre 100-299 estudiantes. En el caso de Quintana Roo, existe variedad en el número de estudiantes, que fluctúa principalmente entre 200-600 estudiantes.

En relación con la ubicación de las escuelas donde trabajan los directores, se encontró que la mayor parte de los participantes de Quintana Roo trabajan en escuelas en zonas urbanas (57\%), mientras que la mayor parte de los directores que participaron de Campeche lo hacen en escuelas en zonas rurales $(53.2 \%)$ y $9.6 \%$ en zonas rurales remotas. En el caso de Yucatán, la mitad de los participantes indicó trabajar para una escuela en la capital y la otra mitad en zona rural.

\section{Instrumento}

Se utilizó un cuestionario que fue diseñado con base en los estudios de caso de la primera etapa del International Study of Principal Preparation (Webber, 2013; Webber y Scott, 2013), el cual ha servido como base para estudios en otros países (Webber, Mentz, Scott, Okoko y Scott, 2014).

El instrumento fue administrado a directores de escuela noveles durante sus tres primeros años de servicio con la intención de determinar los desafíos que encuentran en su trabajo y la medida en que consideran que estaban preparados para enfrentarlos en su puesto.

En relación con los procedimientos, los cuestionarios se enviaron a las escuelas en las que trabajan los directores. Se les dio un mes para responder la encuesta. Una vez que la completaron, la devolvieron en un sobre sellado a la Secretaría de Educación de su estado, donde cada una de ellas fue recogida para su análisis.

Se pidió a los participantes que calificaran los ítems del cuestionario utilizando una escala de cinco puntos: totalmente de acuerdo, de acuerdo, en desacuerdo, totalmente en desacuerdo o no sé. Calificaron primero respondiendo si el ítem representaba un problema en su trabajo y luego indicaron si estaban adecuadamente preparados para resolverlo. 

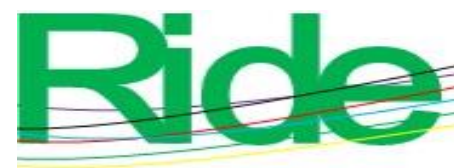

Revista Iberoamericana para la Investigación y el Desarrollo Educativo ISSN 2007 - 7467

Asimismo, el cuestionario incluía tres preguntas abiertas:

1) Por favor, comente sobre cualquier aspecto de su trabajo como director sobre el que le hubiera gustado estar mejor preparado antes de tomar el puesto.

2) Por favor, comente sobre la utilidad de su preparación antes de tomar el puesto, considerando sus experiencias durante sus primeros años como director de escuela.

3) Agregue cualquier comentario, observación o sugerencia que considere pertinente sobre la preparación de los directores de escuela.

\section{Procedimiento}

Los resultados fueron registrados y sometidos a diferentes análisis considerando el tipo de datos. Para los datos cuantitativos, se utilizó el programa estadístico SPSS; para los comentarios, se utilizó un análisis cualitativo, donde se identificaron patrones, temas comunes o categorías, así como un contraste con la teoría.

\section{Resultados}

A continuación, en la figura 1 se presentan las respuestas de los directores de los tres estados en relación con los problemas que enfrentan en sus trabajos. 


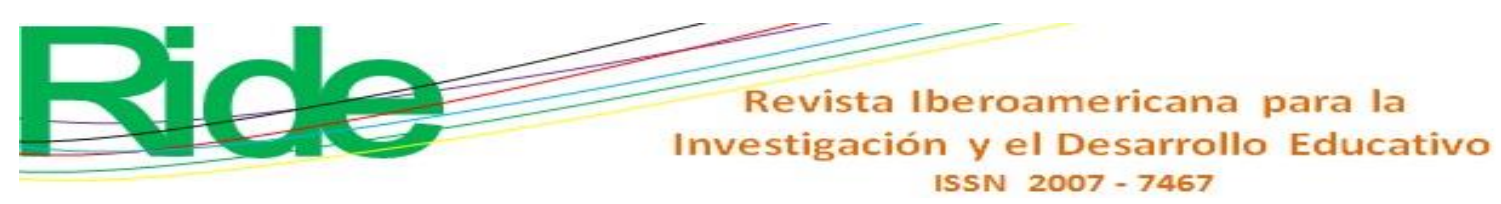

Figura 1. Aspectos problemáticos de los primeros años como director

\begin{tabular}{|c|c|c|c|c|c|}
\hline \multirow{3}{*}{$\begin{array}{l}\text { 10. Entender la cultura de la comunidad en la cual la escuela } \\
\text { está localizada }\end{array}$} & \multicolumn{5}{|c|}{$\|$ Acuerdo $=$ Desacuerdo } \\
\hline & \multicolumn{2}{|c|}{ Yucatán } & \multicolumn{2}{|c|}{ Campeche } & Quintana Roo \\
\hline & $30 \%$ & $69 \%$ & $54 \%$ & $42 \%$ & $34 \% \quad 40 \%$ \\
\hline $\begin{array}{l}\text { 11. Entablar relaciones con la gente de la comunidad donde mi } \\
\text { escuela está localizada }\end{array}$ & $29 \%$ & $67 \%$ & $49 \%$ & $48 \%$ & $29 \% \quad 43 \%$ \\
\hline $\begin{array}{l}\text { 12. Tener credibilidad como director(a) en la comunidad en } \\
\text { donde mi escuela está localizada }\end{array}$ & $29 \%$ & $70 \%$ & $45 \%$ & $49 \%$ & $20 \% \quad 51 \%$ \\
\hline 13. Iniciar proyectos de mejora escolar & $39 \%$ & $61 \%$ & $46 \%$ & $46 \%$ & $34 \%$ \\
\hline 14. Dar continuidad a las iniciativas de mejora escolar & $35 \%$ & $65 \%$ & $46 \%$ & $48 \%$ & $26 \% \quad 40 \%$ \\
\hline $\begin{array}{l}\text { 15. Interactuar con personal docente y administrativo con bajo } \\
\text { desempeño profesional }\end{array}$ & $37 \%$ & $63 \%$ & $42 \%$ & $50 \%$ & $20 \% \quad 51 \%$ \\
\hline 16. Construir relaciones positivas con el personal & $31 \%$ & $70 \%$ & $45 \%$ & $47 \%$ & $17 \% \quad 54 \%$ \\
\hline 17. Mejorar las capacidades profesionales de los profesores & $39 \%$ & $60 \%$ & $48 \%$ & $46 \%$ & $23 \% \quad 49 \%$ \\
\hline 18. Manejar conflictos & $29 \%$ & $70 \%$ & $42 \%$ & $50 \%$ & $46 \%$ \\
\hline $\begin{array}{l}\text { 19. Interactuar con padres de familia para tratar asuntos acerca } \\
\text { de la educación de sus hijos. }\end{array}$ & $30 \%$ & $80 \%$ & $38 \%$ & $51 \%$ & $34 \%$ \\
\hline 20. Organizar mi tiempo & $34 \%$ & $63 \%$ & $42 \%$ & $49 \%$ & $43 \% \quad 29 \%$ \\
\hline 21. Enfrentar la soledad en el puesto & $19 \%$ & $80 \%$ & $36 \%$ & $52 \%$ & $23 \% \quad 46 \%$ \\
\hline 22. Sentirme seguro como líder de escuela & $24 \%$ & $72 \%$ & $45 \%$ & $48 \%$ & $29 \%$ \\
\hline 23. Enfrentar la visibilidad de mi trabajo de manera cotidiana & $26 \%$ & $72 \%$ & $44 \%$ & $50 \%$ & $23 \% \quad 49 \%$ \\
\hline 24. Lograr un equilibrio entre mi vida laboral y personal & $33 \%$ & $67 \%$ & $49 \%$ & $44 \%$ & $34 \%$ \\
\hline 25. Aplicar la normatividad & $37 \%$ & $61 \%$ & $47 \%$ & $44 \%$ & $40 \% \quad 29 \%$ \\
\hline 26. Lidiar con el papeleo administrativo & $40 \%$ & $61 \%$ & $46 \%$ & $49 \%$ & $34 \%$ \\
\hline 27. Tener acceso a las autoridades del sistema escolar & $26 \%$ & $72 \%$ & $38 \%$ & $53 \%$ & $23 \% \quad 49 \%$ \\
\hline $\begin{array}{l}\text { 28. Encontrar un equilibrio entre las demandas del sistema } \\
\text { escolar y las necesidades de la comunidad local }\end{array}$ & $31 \%$ & $67 \%$ & $50 \%$ & $44 \%$ & $43 \% \quad 29 \%$ \\
\hline $\begin{array}{l}\text { 29. Gestionar los recursos apropiados para la operación de la } \\
\text { escuela }\end{array}$ & $26 \%$ & $73 \%$ & $45 \%$ & $48 \%$ & $43 \% \quad 29 \%$ \\
\hline 30. Manejar los recursos financieros de la escuela & $15 \%$ & $83 \%$ & $34 \%$ & $56 \%$ & $29 \%$ \\
\hline 31. Dotar a la escuela de personal eficiente & $30 \%$ & $63 \%$ & $53 \%$ & $39 \%$ & $32 \% \quad 40 \%$ \\
\hline
\end{tabular}

Fuente: Elaboración propia

Tal como se aprecia en la figura 1, Campeche es el estado donde los directores señalaron más aspectos problemáticos. Destaca particularmente entender la cultura de la comunidad en la cual la escuela está localizada, y el dotar a la escuela de personal eficiente. En Yucatán hubo una frecuencia de retos menor. Las más altas fueron el lidiar con el papeleo administrativo, el iniciar proyectos de mejora escolar, y mejorar las capacidades profesionales de los profesores. Finalmente, en Quintana Roo los retos presentaron un comportamiento más heterogéneo, con algunos con altas frecuentes y otras menos identificados. En particular, resalta el poder organizar su tiempo, encontrar un equilibrio entre 


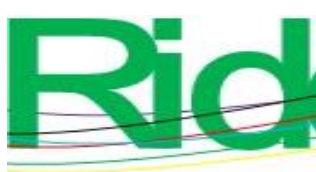

\section{Revista Iberoamericana para la Investigación y el Desarrollo Educativo ISSN 2007 - 7467}

las demandas del sistema escolar y las necesidades de la comunidad local, y gestionar los recursos apropiados para la operación de la escuela.

Figura 2. Aspectos en los que estaba preparado antes de ocupar el puesto de director

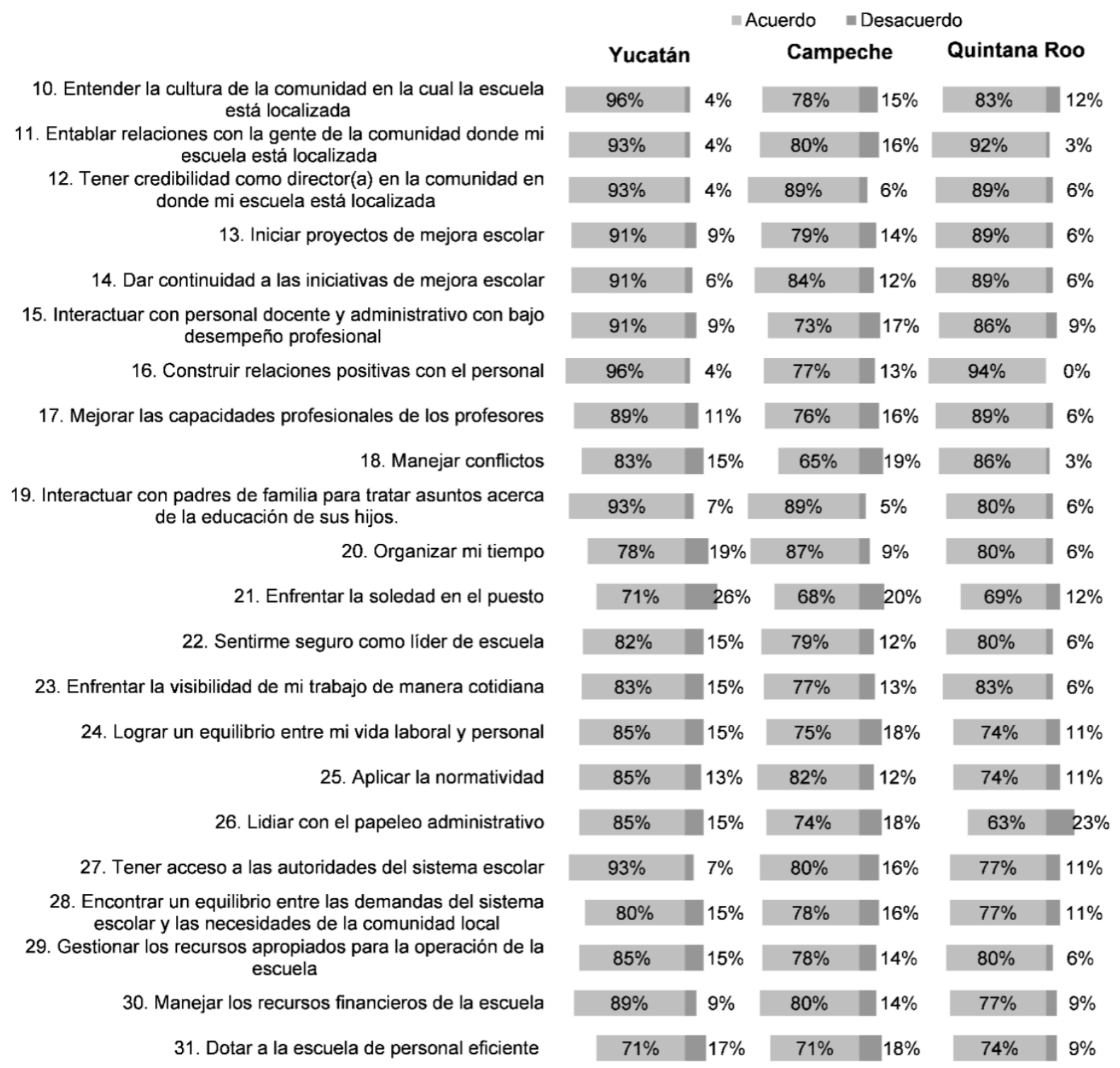

Fuente: Elaboración propia

En general, en la figura 2 se aprecia que los directores usualmente se sienten preparados en la mayoría de los aspectos y funciones de su puesto como director. Aquí resalta que tanto en Yucatán como en Campeche enfrentar la soledad del puesto presentó una mayor problemática en su preparación; mientras que en Quintana Roo se encontró el lidiar con el papeleo administrativo. 


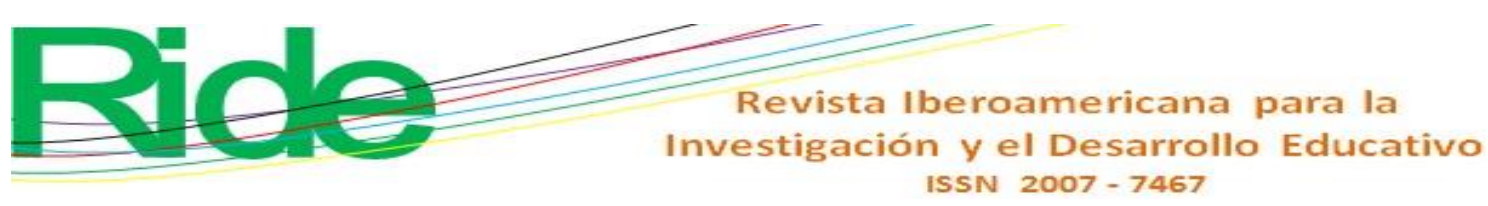

Figura 3. Evaluación de sus experiencias de aprendizaje antes de tomar el cargo de director

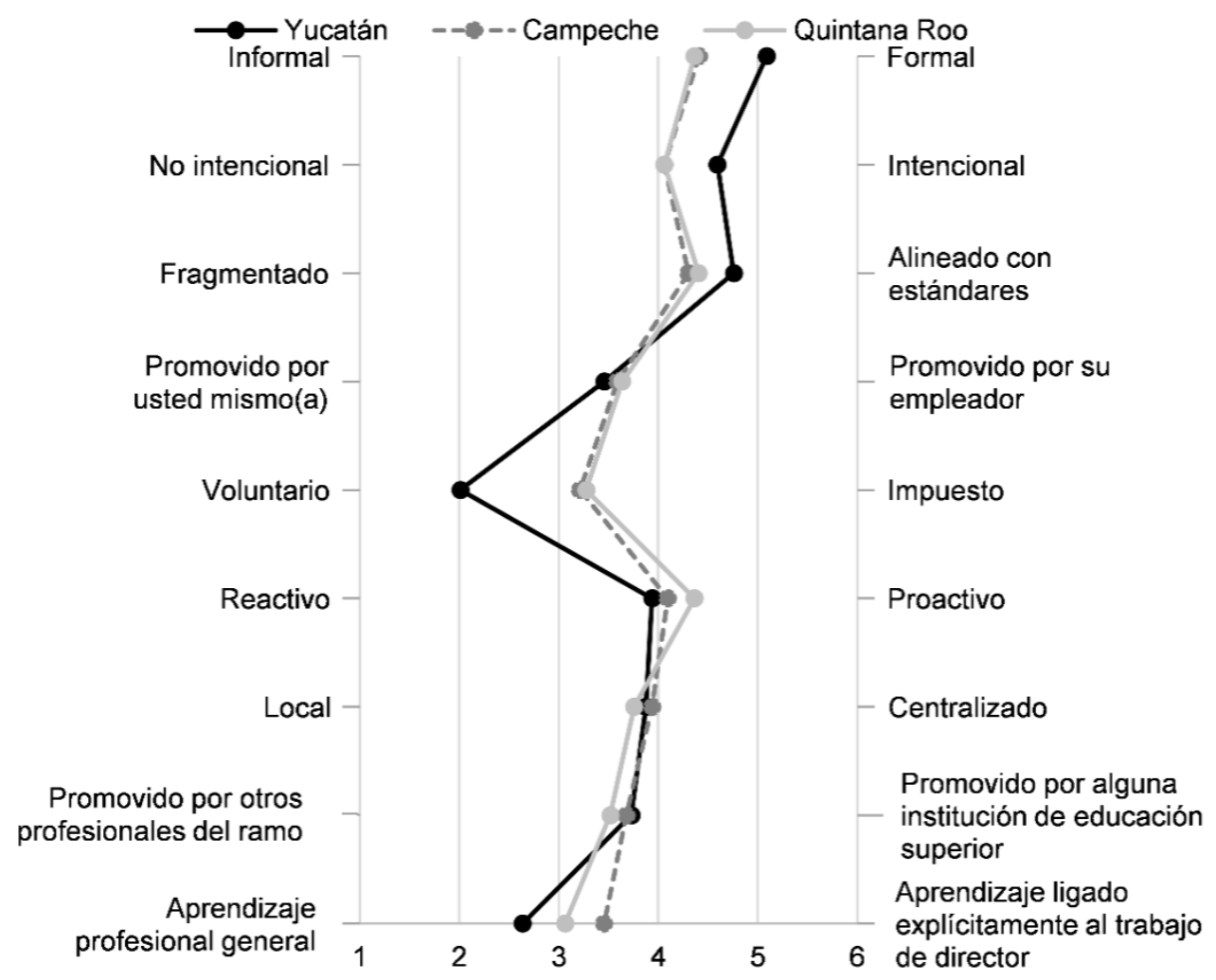

Fuente: Elaboración propia

De manera general, se encontró bastante homogeneidad en los tres estados en sus evaluaciones de sus experiencias de aprendizaje antes de tomar el cargo de director, sin embargo, como se aprecia en la figura 3, Yucatán parece tener una orientación más hacia lo formal, intencional, alineado a los estándares y voluntario que los otros dos estados.

El análisis de los datos indicó que, de forma global, los directores de los tres estados identificaron algunos retos o desafíos de manera similar: aplicar la normatividad y lidiar con el papeleo administrativo (42\%); lograr un equilibrio entre su vida laboral o personal (39\%); mejorar las capacidades de los profesores (38\%); dar continuidad a iniciativas de mejora escolar (36\%); interactuar con personal docente y administrativo con bajo desempeño profesional (34\%); manejar conflictos (32\%), y tener acceso a las autoridades del sistema escolar $(30 \%)$.

En todos los demás problemas o desafíos, se encontraron diferencias significativas entre las respuestas de los directores de Yucatán, Campeche y Quintana Roo.

En relación con las respuestas a las preguntas abiertas, en la primera pregunta los directores indicaron los temas en los que les gustaría estar mejor preparados para su puesto: $24 \%$ mencionó formación en gestión escolar, $20.3 \%$ indicaron la necesidad de más 


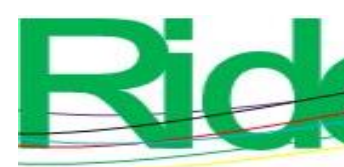

Revista Iberoamericana para la
Investigación y el Desarrollo Educativo
ISSN $2007-7467$

formación sobre reglas, políticas y procedimientos, $13 \%$ sobre pedagogía, psicología y orientación y 12.7 \% sobre tecnologías de información y comunicación.

En respuesta a la segunda pregunta, la mayor parte de los directores dijeron estar adecuadamente preparados en todas las áreas, como se aprecia en el siguiente comentario:

No siento que tenga un problema importante en este puesto porque mi experiencia como maestra me ha apoyado y las cosas se lograron tal como se presentaron. Creo que la disposición más importante es hacer las cosas y no tener miedo. Mi experiencia me ha hecho sentir segura y estoy contenta con mi trabajo

Los directores hablaron positivamente sobre su preparación y experiencia en la docencia. Un director dijo:

(Mi preparación fue) muy útil porque antes de obtener un puesto permanente de director, había sido director interino, y esto me ayudó mucho, y me preparé como docente a lo largo de 26 años de servicio, lo cual considero suficiente experiencia y preparación.

En sus comentarios finales, 13 de los directores sugirieron que cursos adicionales los ayudarían a desarrollarse profesionalmente.

Tres de ellos comentaron:

Sugiero que se asesore y se capacite mejor a los futuros directores para que lleguen a sus puestos con una visión más amplia de cumplimiento de objetivos.

Creo que todos los directores nos beneficiaríamos de participar en cursos, talleres o programas formales de preparación sobre liderazgo; esto complementaría nuestro desarrollo y contribuiría a la mejora de la calidad de la educación

La preparación académica es importante porque permite un conocimiento profundo de los contenidos y otros conocimientos necesarios en la elaboración del trabajo, pero la experiencia es indispensable porque proporciona todos los elementos necesarios para ser un buen líder y el trabajo de director es para ser un buen líder, para crear un clima laboral positivo. 

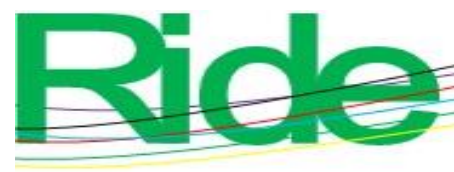

Revista Iberoamericana para la Investigación y el Desarrollo Educativo

ISSN $2007-7467$

Más allá de estas conclusiones directas, puede haber otras cuestiones implícitas. Para comprender mejor esto, es importante notar tres comentarios únicos que iban más allá de las respuestas de la mayoría de los directores. ¿Qué nos dicen las voces de estas personas sobre los desafíos de los directores de escuela?

El primer comentario fue de un director que planteó dudas sobre el sistema y expresó críticas. Se quejó de la falta de compromiso de los supervisores:

Hay que aprender a aceptar la decisión de los jefes, aunque carezcan de fundamento legítimo para apoyarnos, son decisiones personales y egoístas que nos impiden cumplir con la normativa y cumplir con nuestras responsabilidades como directores.

Un segundo director habló de manera más positiva, pero dio a entender que ese liderazgo democrático y un clima positivo pueden faltar en muchas escuelas.

El director siempre debe ejercer liderazgo democrático y promover la colegialidad para permitir la creación de un clima laboral positivo y de satisfacción que se traduzca en productividad y trabajo efectivo como producto de un equipo de liderazgo docente, pero esto no siempre es así.

Un tercer director enfatizó los problemas críticos de la pobreza y la necesidad de conectar la escuela y la comunidad.

Como directora tengo un campo de acción más amplio, y es un desafío porque no solo proveo servicios educativos, sino también a la comunidad en general, y por eso siento las necesidades y deseos que no son atendidos por otras agencias. Como consecuencia, me concentro en los sectores más pobres de la comunidad. Las autoridades no prestan atención a estas necesidades.

Estas tres opiniones dan forma a los problemas identificados por los directores en las respuestas al cuestionario y pueden dar lugar a futuros estudios acerca del contexto y el liderazgo escolar. 


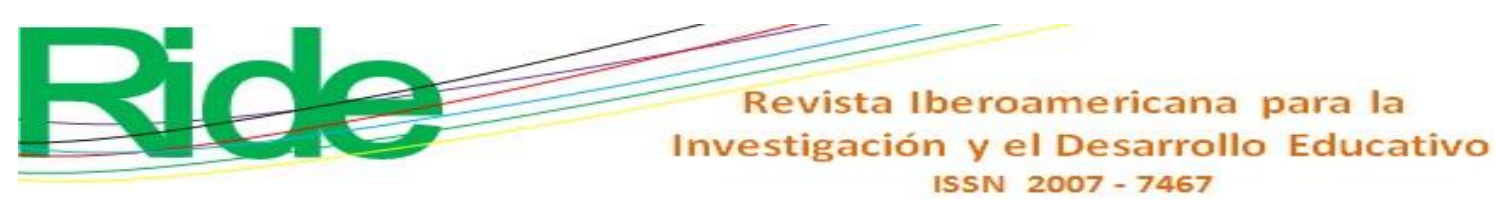

\section{Discusión}

De todo lo anterior, podemos concluir que la mayoría de las personas que ejercen la dirección escolar en las escuelas primarias de los tres estados son hombres. A pesar de que estos son una minoría en el número de graduados en educación, son mayoría en el puesto de dirección. Entre los tres estados, Yucatán fue el estado con mayor número de directoras (41\%), seguido de Quintana Roo (31\%), Campeche fue el estado con el menor número de directoras $(20 \%)$. Estos resultados son consistentes con Lee y Mao (2020), quienes encontraron en su investigación que las mujeres y personas pertenecientes a grupos minoritarios están subrepresentados en puestos de dirección, lo que podría ser evidencia de discriminación en los procesos de selección para la dirección escolar.

En relación con los retos que enfrentan los directores, se encontraron diferencias significativas entre los tres estados. Por este motivo, futuros estudios deben examinar con mayor profundidad el contexto en el que los directores ejercen sus funciones y la influencia de este en su trabajo y su comunidad escolar.

Los resultados tanto de la sección de problemas o desafíos como de las preguntas abiertas indicaron que algunos directores experimentaron problemas comunes para desarrollar o mantener proyectos de mejora escolar, trabajar con el personal con bajo desempeño, mejorar la capacidad de los profesores, manejar conflictos, lograr un equilibrio entre la vida personal y laboral, aplicar la normatividad, administrar el papeleo y tener acceso a las autoridades del sistema. Casi todos los directores expresaron interés en recibir educación continua, particularmente en forma de cursos o talleres.

Estos resultados indican un mayor nivel de satisfacción por parte de los directores que el reportado por los directores que participaron en los estudios de casos previos. Sin embargo, las áreas de desafío son similares a las mencionadas anteriormente. Trabajar con profesores ha sido un tema frecuente en la literatura internacional y el problema del papeleo ha sido examinado en México (Slater, Boone et al., 2005). El desarrollo o mantenimiento de proyectos de mejoramiento escolar también ha sido reconocido en otros estudios, especialmente porque los proyectos implican un papeleo engorroso (Hobson et al., 2003).

Los directores indicaron estar satisfechos con sus experiencias en sus primeros años en sus puestos en Yucatán y se sienten bien preparados a raíz de sus experiencias previas en las escuelas, aunque esta experiencia es principalmente como docentes y no relacionada con la gestión escolar. 


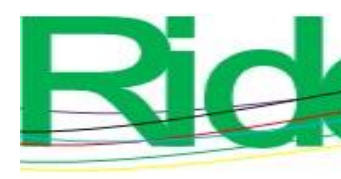

Revista Iberoamericana para la
Investigación y el Desarrollo Educativo
ISSN $2007-7467$

La literatura ha sugerido que el liderazgo debe distribuirse ampliamente (Harris, 2003; Webber y Okoko, 2021), pero el liderazgo en las escuelas mexicanas puede necesitar pasar de un enfoque autocrático a un enfoque democrático, a un liderazgo distribuido. Futuros estudios pueden examinar con mayor profundidad los factores que facilitan o inhiben este tipo de liderazgo en el contexto de la comunidad escolar.

\section{Conclusiones}

A partir de este estudio pudimos concluir varios elementos. Primero, que la dirección escolar sigue siendo un puesto mayormente dominado por el género masculino.

Segundo, es notable cómo la caracterización de los hallazgos pone de manifiesto resultados diferentes para cada estado, por lo que es importante considerar los elementos del contexto para cualquier tipo de estudio orientado a la administración educativa.

Tercero, se encontró que existen desafíos comunes en los directores en sus primeros años, como desarrollar o mantener proyectos de mejora escolar, trabajar con el personal con bajo desempeño, mejorar la capacidad de los profesores, manejar conflictos, lograr un equilibrio entre la vida personal y laboral, aplicar la normatividad, administrar el papeleo y tener acceso a las autoridades del sistema. Estos desafíos pueden ser considerados no solo para su estudio profundo, sino también para el desarrollo de programas de formación y capacitación de futuros directores.

Finalmente, como cuarto punto se considera que, a pesar de los desafíos, la formación previa parece ser percibida favorablemente por los directores participantes, pues, considera, fue de utilidad para sus primeros años de servicio, por lo que se recomienda que los programas de profesionalización para puestos directivos continúen de manera oficial, y se busque orientar nuevas oportunidades de formación en aquellos retos comunes que presentaron los participantes.

\section{Futuras líneas de investigación}

Futuras investigaciones pueden utilizar métodos mixtos para obtener una comprensión más profunda del trabajo de los directores en el contexto del sureste mexicano y en especial del tipo de liderazgo tanto de las autoridades externas a la escuela como dentro del edificio escolar y la manera en que estos liderazgos contribuyen a la democracia y la participación de los diferentes actores. 


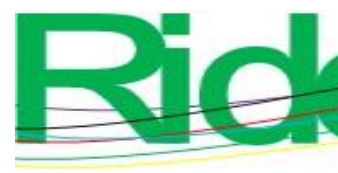

Revista Iberoamericana para la
Investigación y el Desarrollo Educativo
ISSN $2007-7467$

Finalmente, dada la gran participación de participantes hombres en puestos directivos, se recomienda que se considere realizar estudios enfocados de manera específica en aquellas mujeres que desempeñan el puesto de directora en instituciones educativas; es importante establecer estudios que indaguen en las motivaciones de las mujeres directoras, su trayectoria y formación.

\section{Referencias}

Arar, K. (2018). How Novice Principals Face the Challenges of Principalship in the Arab Education System in Israel. Journal of Career Development, 45(6), 580-596. Retrieved from https://doi.org/10.1177/0894845317726392.

Barakat, M., Reames, E. and Kensler, L. A. W. (2019). Leadership Preparation Programs: Preparing Culturally Competent Educational Leaders. Journal of Research on Leadership Education, 14(3), 212-235. Retrieved from https://doi.org/10.1177/1942775118759070.

Bauer, S. C. and Silver, L. (2018). The impact of job isolation on new principals' sense of efficacy, job satisfaction, burnout and persistence. Journal of Educational Administration, 56(3), 315-331. Retrieved from https://doi.org/10.1108/JEA-072017-0078.

Camarillo, J. P. (2006). Factores de éxito y/o fracaso en la implementación del proyecto "La gestión en la escuela primaria”. Revista Electrónica Iberoamericana sobre Calidad, Eficacia y Cambio en Educación, 4(3), 84-107.

Cowie, M. and Crawford, M. (2008). "Being" a new principal in Scotland. Journal of Educational Administration, 46(6), 676-689. Retrieved from https://doi.org/10.1108/09578230810908271.

Day, C., Leithwood, K. and Sammons, P. (2008). What we have learned, what we need to know more about. School Leadership \& Management, 28(1), 83-96. Retrieved from https://doi.org/10.1080/13632430701800102.

Day, C., Sammons, P., Hopkins, D. Leithwood, K. and Kington, A. (2008). Research into the impact of school leadership on pupil outcomes: policy and research contexts. School Leadership \& Management, 28(1), 5-25. Retrieved from https://doi.org/10.1080/13632430701800045. 


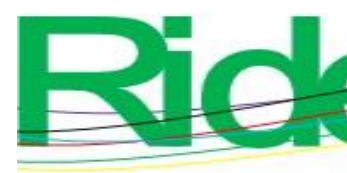

Revista Iberoamericana para la
Investigación y el Desarrollo Educativo
ISSN $2007-7467$

de la Torre, R. (2020). Reporte de movilidad social educativa 2020. Una mirada a las diferencias regionales. Centro de Estudios Espinoza Yglesias.

DeMatthews, D. E., Reyes, P., Carrola, P., Edwards, W. and James, L. (2021). Novice Principal Burnout: Exploring Secondary Trauma, Working Conditions, and Coping Strategies in an Urban District. Leadership and Policy in Schools, 1-17. Retrieved from https://doi.org/10.1080/15700763.2021.1917624.

Gall, M., Gall, J. and Borg, R. (2007). Educational research: An Introduction ( $8^{\text {th }}$ ed.). New York, United States: Pearson Education.

Grissom, J. A., Mitani, H. and Woo, D. S. (2019). Principal Preparation Programs and Principal Outcomes. Educational Administration Quarterly, 55(1), 73-115. Retrieved from https://doi.org/10.1177/0013161X18785865.

Gu, Q., Sammons, P. and Mehta, P. (2008). Leadership characteristics and practices in schools with different effectiveness and improvement profiles. School Leadership \& Management, 28(1), 43-63. Retrieved from https://doi.org/10.1080/13632430701800078.

Hallinger, P. and Heck, R. (1998). Exploring the Principal's Contribution to School Effectiveness: 1980-1995. School Effectiveness and School Improvement, 9(2), 157191. Retrieved from https://doi.org/10.1080/0924345980090203.

Harris, A. (2003). Teacher Leadership as Distributed Leadership: Heresy, Fantasy or Possibility? School Leadership and Management, 23(3), 313-324. Retrieved from https://doi.org/10.1080/1363243032000112801.

Hobson, A., Brown, E., Ashby, P., Keys, W., Sharp, C. and Benefield, P. (2003). Issues for Early Headship. Problems and Support Strategies. United Kingdom: National College for School Leadership.

Karstanje, P. and Webber, C. F. (2008) Programs for school principal preparation in East Europe. Journal of Educational Administration, 46(6), 739-751. Retrieved from https://doi.org/10.1108/09578230810908325.

Kılınç, A. Ç. and Gümüş, S. (2021). What do we know about novice school principals? A systematic review of existing international literature. Educational Management Administration \& Leadership, 49(1), 54-75. Retrieved from https://doi.org/10.1177/1741143219898483. 


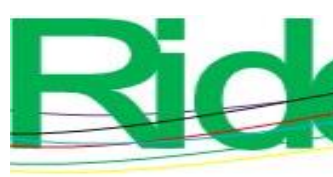

Revista Iberoamericana para la Investigación y el Desarrollo Educativo ISSN $2007-7467$

Lee, S. W. and Mao, X. (2020). Recruitment and selection of principals: A systematic review. Educational Management Administration \& Leadership. Retrieved from https://doi.org/10.1177/1741143220969694.

Leithwood, K. and Day, C. (2008). The impact of school leadership on pupil outcomes. School Leadership \& Management, 28(1), 1-4. Retrieved from https://doi.org/10.1080/13632430701799718.

Leithwood, K., Harris, A. and Hopkins, D. (2008). Seven strong claims about successful school leadership. School Leadership \& Management, 28(1), 27-42. Retrieved from https://doi.org/10.1080/13632430701800060.

Leiva, M. V., Montecinos, C. y Aravena, F. (2016). Liderazgo pedagógico en directores nóveles en Chile: Prácticas de observación de clases y retroalimentación a profesores. Revista Electrónica de Investigación y Evaluación Educativa, 22(2). Recuperado de https://doi.org/10.7203/relieve.22.2.9459.

Loera, A., Hernández, R. y García, E. (2006). Buenas prácticas de gestión escolar y participación social en las escuelas PEC. Resultados de la comparación de muestras polarizadas por niveles de logro y eficacia escolar 2001-2006. Chihuahua, México: Heurística educativa.

Marshall, I. (2018). Novice Principals Speak Out: A Qualitative Analysis of the Transition from Teacher to Principal in Barbadian Primary School. Caribbean Journal of Education, 40(1\&2), 139-169.

Meyer, F., Sinnema, C and Patuawa, J. (2019). Novice principals setting goals for school improvement in New Zealand. School Leadership and Management, 39(2), 198-221. Retrieved from https://doi.org/10.1080/13632434.2018.1473358.

Muñoz, M., Murillo, F. J., Barrio, R., Brioso, M. J. Hernández, M. L. y Pérez, M. J. (2000). La mejora de la eficacia escolar: un estudio de casos. España: Ministerio de Educación, Cultura y Deporte.

Murillo, F. J., Barrio, R. y Pérez, M. J. (1999). La dirección escolar. Análisis e investigación. Madrid, España: Ministerio de Educación y Cultura, Centro de Investigación y Documentación Educativa.

Murphy, G. (2020). Leadership preparation, career pathways and the policy context: Irish novice principals' perceptions of their experiences. Educational Management Administration \& Leadership, 1-22. Retrieved from https://doi.org/10.1177/1741143220968169. 


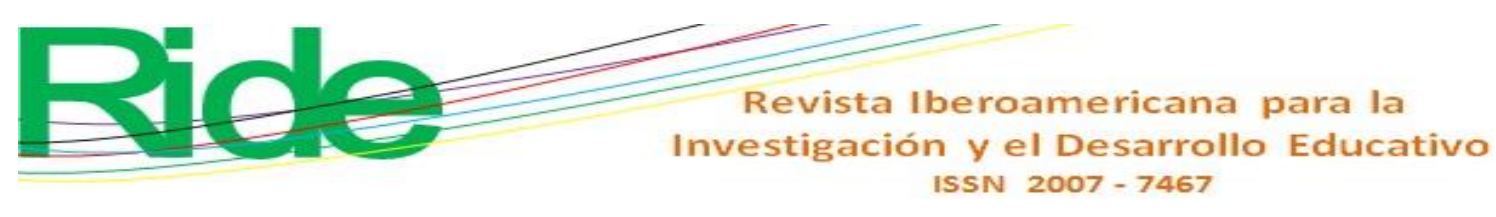

Nelson, S. W., de la Colina, M. and Boone, M. D. (2008). Lifeworld or systemsworld: what guides novice principals? Journal of Educational Administration, 46(6), 690-701. Retrieved from https://doi.org/10.1108/09578230810908280.

Onguko, B., Abdalla, M. and Webber, C. F. (2012). Walking in unfamiliar territory: Headteachers' preparation and experiences in their first year in Tanzania. Educational Administration Quarterly, 48(1), 86-115. Retrieved from https://doi.org/10.1177/0013161X11423391.

Onguko, B., Abdalla, M. and Webber, C. F. (2008). Mapping principal preparation in Kenya and Tanzania. Journal of Educational Administration, 46(6), 715-726. Retrieved from https://doi.org/10.1108/09578230810908307.

Oplatka, I. and Lapidot, A. (2018). Novice principals' perceptions of their mentoring process in early career stage: the key role of mentor-protégé relations. Journal of Educational Administration and History, 50(3), 204-222. Retrieved from https://doi.org/10.1080/00220620.2017.1420044.

Prado, A. and Spillane, J. P. (2019). Novice School Principals Constructing Their Role VisÀ-Vis External Stakeholders: (Not) Attempting to Be "All Things to All People". Educational Administration Quarterly, 55(5), 812-840. Retrieved from https://doi.org/10.1177/0013161X18822101.

Rice, J. K. (2010). Principal Effectiveness and Leadership in an Era of Accountability: What Research Says. Washington, United States: National Center for Analysis of Longitudinal Data in Education Research.

Ruiz, G. (1999). Un acercamiento a la calidad de la educación primaria en Aguascalientes desde la perspectiva de la efectividad escolar. Aguascalientes, México: Universidad Autónoma de Aguascalientes.

Sandoval, L. Y., Pineda, C., Bernal, M. y Quiroga, C. (2019). Los retos del director escolar novel: formación inicial y liderazgo. Revista Complutense de Educación, 31(1), 117126. Recuperado de https://doi.org/10.5209/rced.61919.

Scott, S. and Webber, C. F. (2008). Evidence-Based Leadership Development: The 4L Framework. Journal of Educational Administration, 46(6), 772-776. Retrieved from https://eric.ed.gov/?id=EJ817192.

Secretaría de Educación Pública [SEP]. (1999). El proyecto escolar: una estrategia para transformar nuestra escuela. México: Secretaría de Educación Pública. 


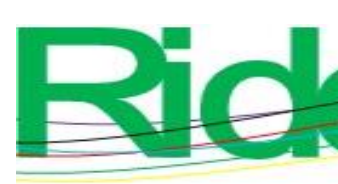

Revista Iberoamericana para la
Investigación y el Desarrollo Educativo
ISSN $2007-7467$

Swen, C. P. (2020). Talk of Calling: Novice School Principals Narrating Destiny, Duty, and Fulfillment in Work. Educational Administration Quarterly, 56(2), 177-219. Retrieved from https://doi.org/10.1177/0013161X19840387.

Tahir, L., Thakib, M. T. M., Hamzah, M. H., Said, M. N. H. M. and Musah, M. B. (2017). Novice head teachers' isolation and loneliness experiences: A mixed-methods study. Educational Management Administration \& Leadership, 45(1), 164-189. Retrieved from https://doi.org/10.1177/1741143215587302.

Viloria, M., Volpe, T., Guajardo, R. A. and Kyle, R. (2021). Novice school principals' advice to educational administration students. Journal of Latinos and Education, 20(2), 136147. Retrieved from https://doi.org/10.1080/15348431.2019.1568251.

Webber, C. F. (2008). Principal preparation: international perspectives. Journal of Educational Administration, 46(6). 673-675. Retrieved from https://doi.org/10.1108/jea.2008.07446faa.001.

Webber, C. F. (2013). Template versus awareness. In Slater, C. L. and Nelson, S. (eds.), Understanding the Principalship: An International Guide to Principal Preparation (pp. 71-94). Bingley, United Kingdom: Emerald.

Webber, C. F. and Okoko, J. M. (2021). Exploring Teacher Leadership Across Cultures: Introduction to Teacher Leadership. Research in Educational Administration \& Leadership, 6(1), 1-15.

Webber, C. F. and Scott, S. (2013). Principles for principal preparation. In Slater, C. L. and Nelson, S. (eds.), Understanding the Principalship: An International Guide to Principal Preparation (pp. 95-122). Bingley, United Kingdom: Emerald.

Webber, C. F., Mentz, K., Scott, S., Okoko, J. M. and Scott, D. E. (2014). Principal preparation in Kenya, South Africa, and Canada. Journal of Organizational Change Management, 27(3), 499-519. Retrieved from https://doi.org/10.1108/JOCM-072013-0125.

Wildy, H. and Clarke, S. (2008). Principals on L-plates: rear view mirror reflections. Journal of Educational Administration, 46(6), 727-738. Retrieved from https://doi.org/10.1108/09578230810908316. 